\title{
« Je ne suis pas écrivain suisse ». William Ritter, la Suisse et sa littérature
}

\author{
Denis Bussard \\ Archives littéraires suisses
}

\begin{abstract}
Ritter, mais aussi de sa correspondance, et des recensions de ses deux premiers romans, cette contribution se propose de revenir sur la place occupée par le jeune écrivain neuchâtelois au sein du champ littéraire romand de la fin du XIX ${ }^{e}$ siècle. Les modèles symbolistes et décadents choisis par Ritter, de même que le style et l'intrigue d'Ægyptiacque (I89I) ou d'Âmes blanches (I893) le placeront à la marge de la littérature produite alors en Suisse - littérature dont il critique le manque d'art et l'inspiration protestante. Tache aveugle de l'histoire de la littérature, l'œuvre (décadente) du jeune Ritter a peut-être pu, dans une certaine mesure, préparer le terrain aux avant-gardes romandes du début du $\mathrm{xx}^{\mathrm{e}}$ siècle, ne serait-ce que dans l'émancipation de la morale et l'attention magnifiée à la langue.
\end{abstract}

Keywords: William Ritter, histoire littéraire, Suisse romande, décadence

« Je ne suis pas écrivain suisse, trop à l'écart de tout et de tous, par toutes ces contradictions : mon catholicisme féodal, mon culte de la passion, mon amour de la splendeur de la chair, et ma haine tout à la fois du "grimpionnat" et des titres mal portés, du militarisme d'aujourd'huy et du prolétarisme de demain, pour être jamais l'aîné de personne entre Alpes et Jura, et je broie mon noir à l'écart, même de tout ce que j'aime en ce qui fut "canton de Sarine et Broye" ».

Si William Ritter a rompu très tôt les amarres avec son pays natal, « dévoré d'une frénésie d'en allade sur les grand' routes $»^{2}$, le caractère définitif du propos semble bien marquer la fin d'un cycle amorcé dès les années I880-I890 et la parution de ses premiers romans, Ægyptiacque (Savine I89I) puis Âmes blanches (Lemerre I893), qui ont tous deux été rédigés en Suisse et qui se déroulent à Neuchâtel. Admettant une forme de nostalgie à l'égard de Fribourg, mais refusant tour à tour les étiquettes comme les filiations littéraires, Ritter se veut résolument en marge, de la Suisse comme de ses compatriotes. Fréquemment associé, dans la littérature secondaire, aux cercles décadents et à l'esthétique fin-de-siècle, il semble effectivement faire figure de météorite dans le paysage culturel romand de l'époque. Cette contribution vise donc à interroger la place occupée en Suisse par le Neuchâtelois

I Lettre autographe signée (l.a.s.) de William Ritter (WR) à Gonzague de Reynold (GdR), Munich, 12.06.1903, Fonds Reynold, corr. aut. I84, ALS, Berne.

2 L.a.s. de WR à GdR, Munich, 28.Io.I905, Fonds Reynold, corr. aut. I84, ALS, Berne. 
au tournant du siècle, en rappelant non seulement les discours produits par Ritter sur les écrivains contemporains et sur la littérature telle qu'elle était conçue et promue en Suisse francophone, mais aussi en se penchant sur la réception de son œuvre par la critique et sur l'accueil de ses textes au sein des organes de presse suisses ${ }^{3}$.

\section{«Il y a beau temps que, en fait de lectures, je me suis mis hors la loi commune $»^{4}$. William Ritter critique littéraire}

Connu surtout pour ses chroniques artistiques et pour ses études musicologiques, Ritter l'est beaucoup moins pour les écrits qu'il a publiés sur ses confrères écrivains. Outre le jugement de valeur plutôt négatif que l'on peut porter sur son œuvre de critique littéraire (Donzé I999:45), il faut relever que celle-ci fut moins abondante en effet. À notre connaissance, Ritter n'a pas eu le loisir de tenir une chronique littéraire de manière prolongée et régulière dans un périodique, à l'exception du travail de bibliographe qu'il a mené dans les années 1890 à La Liberté de Fribourg et au National suisse neuchâtelois, puis pour le compte du Magasin littéraire et scientifique de Gand entre décembre I896 et août I898. La critique littéraire "ritterienne" fut aussi moins systématique dans la mesure où il n'a guère pris la peine de rédiger un texte programmatique. On peut toutefois déceler quelques invariants, qu'il expose dans la première livraison de sa « Revue des livres, des estampes et de la musique-publiée » au Magasin littéraire :

Je me croirai absolument tenu de dire la vérité et toute la vérité à ceux qui me la demandent [...]. Je m'expose donc à un double danger : n'être pas toujours agréable aux auteurs, et parfois être désagréable à mes lecteurs aussi en leur parlant de choses ou de gens dont ils aimeraient mieux ne rien savoir, quand bien même j'en dirai ce qu'un catholique doit en dire. Je tâcherai de me le faire pardonner par un grand zèle à lire, voir et jouer les œuvres des inconnus et des méconnus, par un grand respect chez les arrivés de tout effort désintéressé et de toute noble conviction [...]. Je ne cache pas, en outre, ma tendresse particulière pour les jeunes et les hardis ; elle n'ira jamais jusqu'à l'irrévérence gratuite envers les devanciers. [...] En un mot, je ferai de mon

\footnotetext{
3 En l'absence de bibliographie exhaustive des écrits de Ritter, on se basera, pour cette étude, sur trois sources : les textes disponibles sur les plateformes numériques (Gallica-BnF pour les revues françaises, la Digithèque des Bibliothèques de l'ULB pour les périodiques belges, Scriptorium et les archives de la Gazette de Lausanne, de L'Impartial, de La Liberté pour la Suisse) ; les 592 articles publiés entre I886 et I96I, recensés et conservés par la Bibliothèque de la Ville de La Chaux-de-Fonds qui les a acquis en 1966 ; le "Catalogue de l'œuvre publiée » établi par Josef Ritter-Tcherv et conservé aux ALS.

4 WR, «Causerie littéraire. Livres récents ", in Le National suisse (à propos du Satanisme et la Magie de Jules Bois), 22.II.I895.
} 
mieux, trop heureux si ces notes me valent un ou deux amis, autant que possible parmi ceux à qui la vie, le public et les éditeurs sont très durs, le rêve très consolateur et qui ont très profond le respect de leur chimère ${ }^{5}$.

Défense de l'idéal contre le réalisme et le matérialisme, promotion des œuvres catholiques et des jeunes écrivains, sincérité du propos comme de la critique, telle paraît être la ligne de conduite de Ritter - pas très éloignée, à certains égards, du programme artistique énoncé en tête du premier volume de L'Art en Suisse (Arnold Böcklin, 1895) dont il fait une « œuvre de combativité et de polémique pour le beau, en même temps qu'histoire partiale de [ses] admirations et de [ses] haines déterminées par des principes impartiaux». Ces quelques «principes » sont égrenés surtout au fil de ses chroniques. La Nichina de Hugues Rebell est ainsi l'occasion de rappeler la parfaite adéquation entre art et religion catholique (" Je veux bien qu'il soit dit, et le répéter est une des fonctions de ma vie, que l'essence de l'art est catholique, que le Catholicisme renferme tout ce qu'il y a de plus haut et idéal dans l'art $\left.{ }^{6}\right)$. Les poèmes de Stuart Merrill ou de Max Elskamp lui permettent d'insister sur la sincérité et le dépouillement nécessaires à la connaissance de l'homme véritable (Merrill est certes " habile", "mais l'homme que je cherche partout, où est-il ? Que pense-t-il de la vie, de lui-même, à quel demain tendil ? », quant au recueil de Elskamp, La Louange de la vie, Ritter s'interroge : « Me renseigne-t-il sur l'âme de Max Elskamp, puis-je d'après [lui] me le représenter, m'en faire un ami, ma question de toujours en ouvrant un livre de poète $»^{8}$ ). L'inscription d'une œuvre dans un contexte géographique et temporel donné est réaffirmée sous la forme d'une critique adressée à Dilettantes d'Emmy de Némethy («quand on a le bonheur de n'être pas né à Paris, qu'on aime si fort un pays aussi beau, aussi orgueilleux, et malgré tout encore aussi mal connu que la Hongrie, [...] pourquoi écrire un roman parisien ? $»^{9}$ ) et à Villa sans maitre de Georges Rouart ("Les livres abstraits qui se veulent hors d'un temps et d'un espace trop déterminés ne sont pourtant pas mon fort $\left.\aleph^{\mathrm{I}}\right)$. Les Déracinés de Maurice Barrès sont un prétexte pour dénoncer le naturalisme zolien (« Qui doit rager de son livre, c'est Zola ! Car pour le faire rentrer dans sa bauge, lui avec ses malpropres rinçures de prétendue science, le voilà le vrai roman réaliste $\left.{ }^{\mathrm{II}}\right)$, et Sainte Marie des Fleurs de René

5 WR, "Revue des livres, des estampes et de la musique-publiée ", Le Magasin littéraire et scientifique, 15.12.1896, pp. 372-373.

6 Ibidem, 15.07.1897, p. I3.

7 Ibidem, I5.I2.1897, p. 339 .

8 Ibidem, I5.03-I5.04.I898, p. 225.

9 WR, «Causerie littéraire. Livres récents », Le National suisse, o8.II.I895.

Io WR, «Revue des livres, des estampes et de la musique-publiée », Le Magasin littéraire et scientifique, 15.07-15.08.1898, p. I0.

II Ibidem, I5.03-I5.04.1898, p. 205. 
Boylesve de critiquer le matérialisme et le positivisme petit-bourgeois ( il pouvait être grandiose, s'il avait mieux exprimé tout le réalisme mécanique, politique, nietschien, utilitaire du présent, étranglant le passé et l'idéalisme pleins de cœur, de sentiment, de rêve et de poésie, l'Amérique aux prises avec l'Europe $»^{12}$ ).

À ces critères de jugement répondent, comme en miroir, quelques affinités électives. On peut ainsi distinguer les écrivains dont il parle beaucoup et dont il loue les œuvres (non sans faire preuve parfois de réserves, comme Boylesve, Kahn, Mazel, Rambosson, Rebell), des auteurs auxquels il voue un « culte » et qu'il admire (quasiment) sans condition : Barbey d'Aurevilly, qui « représente l'idéal de l'écrivain artiste catholique en France ${ }^{13}$; Péladan, auteur vivant qui a eu le plus d'influence sur la jeune génération ${ }^{14}$; Maeterlinck, « dont [il voudrait] ne jamais parler à personne afin de [se] le réserver à [lui] tout seul $\aleph^{15}$; enfin Barrès (celui des Déracinés en particulier) et Daudet (pour L'Astre noir). Le Panthéon de Ritter est ainsi composé de poètes symbolistes, de romanciers décadents, de chantres du nationalisme et de l'antisémitisme, de partisans monarchistes et de fervents catholiques. De quoi mettre Ritter en porte-à-faux avec la « littérature suisse » de l'époque contre laquelle il rompit quelques lances.

\section{« Je connais une littérature française, une allemande, une italienne et même une romanche, mais pas une littérature suisse ${ }^{16}$. William Ritter contre la littérature « suissarde »}

Né en I867, Ritter publie ses premiers textes en I880-I890, alors que le champ littéraire romand achève son processus d'autonomisation vis-à-vis du centre parisien en définissant des critères de légitimation et de consécration internes, et en offrant un réseau dense de maisons d'édition et de périodiques propres à accueillir les plumes locales. Mais le "parfait" écrivain romand doit suivre un cahier des charges relativement précis : les sujets d'inspiration patriotique sont privilégiés (en pleine fièvre nationaliste européenne, il s'agit alors d'affirmer les mythes identitaires helvétiques, comme la démocratie, la liberté, l'indifférenciation sociale) ; l'art pour l'art est banni et l'œuvre littéraire doit être édifiante et soumise à la morale ; enfin, on se tiendra à bonne distance des avant-gardes françaises (naturalisme, symbolisme) et des recherches esthétiques trop complexes, l'auteur

I2 Ibidem, I5.II.I897, p. 291 .

I3 Ibidem, I5.I2.I896, p. 373.

I4 Ibidem, I5.05.I897, p. 288.

I5 WR, «Livres récents ", Le National suisse, 03.04.1896.

I6 WR, «Enquête sur l'art et la littérature suisses ", La Voile latine, tome II, n I, hiver I906, p. 96. 
romand, pour être compris de tous, devant faire usage d'un langage simple, voire simplifié, gage d'authenticité. La génération à laquelle appartient Ritter ne se reconnaît plus dans ces critères forgés surtout par leurs aînés à partir de 1850 contre la littérature venue de Paris : à la recherche d'expressions nouvelles, épris d'art et de lettres, les jeunes écrivains déplorent l'insuffisance artistique d'une production moralisatrice fort abondante. Selon Ritter, la littérature " suisse », qualifiée alors de "suissarde », ne peut ainsi que désigner « cette production toute spéciale à nos cantons protestants qui est la plus écœurante potion que je connaisse, un vomitif sucré... ». Le terme hautement péjoratif « suissard », utilisé ici en 1906 en réponse à l'enquête de La Voile latine, apparaît de manière précoce, régulière et dans divers contextes sous la plume de Ritter : on le trouve en I898 pour parler d'André Gladès, " dépouillée de tous les prêchiprêcha suissards ${ }^{17}$; dans un texte de fiction, Leurs lys et leurs roses en I903, pour décrire les "idées tricoteuses et suissardes » de la servante aux « vues bourgeoises » et à la "fierté républicaine »; ou encore, en I934, dans sa préface aux «Lettres écrites de Castel Pelesch » envoyées de Roumanie par Léopold Bachelin en I890. Désignant dans un premier temps « un genre de raillerie que [Bachelin] croyait désinvolte et n'était que provincial », le terme acquiert ensuite une signification plus socio-historique, liée au multiculturalisme helvétique. La correspondance de Bachelin sent ainsi le « petit Bernois, frotté de lettres et de bonnes manières neuchâteloises » et constitue, à ce titre, un excellent exemple d'un « document de notre parler... savant d'alors, et que l'on pourrait vraiment appeler un morceau d'architecture rustique. Non pas provincial, mais universitaire allemand dévié en français ${ }^{18}$. Les attaques de Ritter concernent donc autant la morale protestante (dont il fustige « l'erreur, la tournure d'esprit, la mesquinerie, la froideur et l'intolérance ${ }^{19}$ ) que le manque d'art de la production livresque de ce « pays de lourdauds littéraires où les moindres gratte-papiers affectent des grâces d'éléphants bateleurs $»^{20}$. C'est bien une forme d'hypercorrection intériorisée par l'écrivain romand, de classicisme de façade et d'imitation que déplore Ritter en se moquant de la littérature romande qui se veut « si classique, qu'elle ne le sera jamais $»^{21}$. "Tout écrivain suisse ou bien se range et rentre dans la tourbe commune, ou bien courageusement renie et méprisamment oublie l'insigne mesquinerie des siens et s'exile volontairement » : le départ ou la compromission, telles paraissent

I7 WR, « Revue des livres, des estampes et de la musique-publiée », Le Magasin littéraire et scientifique, 15.07-15.08.1898, p. I2.

I8 WR, «Préface » aux « Lettres écrites de Castel Pelesch », Fonds Ritter, ALS, Berne, pp. 7, 24-25.

I9 WR, "Revue des livres, des estampes et de la musique publiée ", Le Magasin littéraire et scientifique, I5.07.1897, p. I2.

20 WR, «Un poète suisse. Louis Duchosal », La Plume, oi.07.1892, n 77, p. 299.

2I WR, « M. Louis Duchosal », La Liberté, 04.04.I89I, p. 3. 
donc être les deux seules alternatives offertes à celui qui fait preuve « d'une première étincelle d'individualité $»^{22}$, déclare-t-il.

Si Ritter consacre bien quelques chroniques littéraires à des compatriotes (Auguste Bachelin, Walter Biolley, Victor Cherbuliez, André Gladès, Valentin Grandjean, Gottfried Keller, Urbain Olivier, Eugène Rambert, Ernest Tissot), il est significatif de relever le nom des trois écrivains de nationalité suisse dont il se sent le plus proche, comme en témoignent les correspondances échangées et les recensions qu'il leur a consacrées. Édouard Rod (I857-I9IO), en particulier pour sa Course à la mort qui « avait fondé [son] admiration $»^{23}$ et dont on a fait un roman décadent ; Léopold Bachelin (I857I930), " cet audacieux qui affichait le plus carabiné des mépris pour un tas de choses, auxquelles il ne fait pas bon toucher à Neuchâtel : la Vénérable Classe, la très haute, très noble, très ancienne et très puissante aristocratie de l'endroit, La Suisse libérale, la peinture des Meuron, la critique des Godet ${ }^{24}$, et qui publia des vers d'un " athéisme panthéiste ", fut proche du parti radical, inventa la critique d'art à Neuchâtel, et usa de « quelques néologismes "décadents" " ${ }^{25}$; Louis Duchosal (I862-I90I), le « symboliste suisse ${ }^{26}$, auteur du Livre de Thulé et fondateur de La Revue de Genève en I885 qui, selon Ritter, « eut tous les vices, toutes les impertinences : wagnérienne, impressionniste, symboliste, catholique même, [La Revue de Genève] bafouait toutes les opinions courantes des Alpes au Jura, du Rhône à la Thièle $\aleph^{27}$. Trois écrivains à l'esthétique fort éloignée de la doxa romande de l'époque et qui connurent des parcours erratiques : le jeune Rod quitta la Suisse pour rejoindre Paris et les cercles naturalistes en I879; Bachelin gagna la Roumanie et entra au service du Roi, en tant que bibliothécaire ; quant à Duchosal, désireux de rejoindre Paris, il dut rester dans la Cité de Calvin, atteint de la maladie de Friedreich : «J'achève de m'éteindre dans un milieu terne. Ah ! si j’avais des jambes! J'irais à Paris, et tous ces jeunes fiévreux verraient ce que peut un Genevois qui a quelque chose dans le ventre et les nerfs en feu $»^{28}$.

22 WR, «Un poète suisse. Louis Duchosal », op. cit.

23 L.a.s. de WR à Édouard Rod, Paris, 30.04.I893, Fonds Rod, IS 599, BCU, Lausanne.

24 WR, «Léo Bachelin », Les Pages littéraires, année 2, n 5-6, I895, pp. 198-207.

25 WR, «Préface » aux «Lettres écrites de Castel Pelesch », Fonds Ritter, ALS, Berne, p. 4.

26 Titre de l'article («Un symboliste suisse ») publié par Léopold Bachelin dans Le Magasin littéraire et scientifique, I5.03.I891, pp. 253-258.

27 WR, «Un poète suisse. Louis Duchosal », op. cit.

28 L.a.s. de Louis Duchosal à Édouard Rod, [Genève], 30.09.I885, Correspondance Louis Duchosal, CH BGE Ms. fr. 2983, f. 30, Genève. 


\section{"La vie en Suisse a été rendue impossible à certains d'entre nous $»^{29}$. William Ritter et la «Suissardie»}

L'opinion que Ritter se forge de la littérature produite en Suisse romande semble indissociable de l'appréciation générale qu'il se fait de son pays natal. Et si l'écrivain a rédigé des pages pleines de douceur à propos de son enfance et donné des descriptions du lac de Neuchâtel qui récoltent même les suffrages de ses plus ardents contradicteurs, il n'a pourtant pas de mots assez durs pour blâmer le pays dans lequel il affirme être né par erreur. En juillet I892, dans une lettre à Duchosal, il dresse ainsi un véritable réquisitoire contre la Suisse, qu'il juge liberticide à plusieurs égards : sont évoquées tour à tour la liberté religieuse (l'exil de dix ans réservé à l'évêque Gaspard Mermillod par le gouvernement radical genevois lors de la nomination du prélat au poste de vicaire apostolique de la Cité de Calvin en 1873), la liberté scientifique (illustrée par le conflit ayant opposé Raoul Pictet, physicien, à l'ingénieur Théodore Turrettini à propos de la gestion d'une société active dans les procédés de congélation) et la liberté artistique (« La Nuit» de Hodler, dont il est un fervent admirateur, ayant dû être retirée du Musée Rath sous prétexte de respecter la bienséance et la moralité publique). Autant d'« affaires » qu'il menace d'utiliser dans une polémique «sans fin » où il se permettrait «sans le moindre scrupule des massacres comme il n'en a point été vu en Suisse depuis la bataille de St-Jacques sur Birse $»^{30}$. Plusieurs cas sont encore évoqués, dont certains concernent plus directement Ritter, sous le triple angle de l'histoire personnelle, familiale et littéraire : les difficultés rencontrées à Neuchâtel par Bachelin, son professeur de littérature grecque et française, et celles que dû affronter son père, Guillaume Ritter (I835-I9I2). Les nombreuses désillusions professionnelles de "l'ingénieur entreprenant » du barrage de la Maigrauge à Fribourg devenu « simple marchand de pierres » à la carrière des Saars ont profondément marqué le jeune William : le déménagement à Neuchâtel « coïncidait avec le brusque passage de la majorité catholique et de [leur] situation enviée de Fribourg à la minorité catholique et à l'humiliation de [leur] exode $»^{31}$. À ces difficultés familiales s'ajoutent ses «affaires personnelles » comme il les nomme. L'interdit qui empêche Ritter de vivre son homosexualité en fait très certainement partie, et cette situation n'est probablement pas étrangère aux sentiments haineux qu'il éprouve vis-à-vis de la société suisse de l'époque (il confie par exemple à Reynold en 1903 sa peine consécutive à la séparation d'avec Mario Segantini, que « Neuchâtel n'a eu ni trêve ni repos avant d'avoir détaché de [lui] »

29 L.a.s. de WR à Louis Duchosal, Besançon, 2I.07.I892, Correspondance Louis Duchosal, CH BGE Ms. fr. 2989, f. 27I, Genève.

30 Ibidem.

3I WR, D’Autrefois, Neuchâtel, Attinger Frères éditeurs, I9I4, pp. 86 et 95. 
et évoque ces nouveaux " pays qui [lui] ont été douceur, refuge et clémence et où [son] cœur n'a jamais été piétiné avec rage froide et géhenné avec un art si recherché, si rectiligne, si calviniste $»^{32}$ ). Mais il y est aussi sûrement question de difficultés plus littéraires - liées à l'accueil qui fut réservé à ses premiers textes et à la place qu'il occupa dans le champ culturel romand de l'époque - qui le poussèrent à quitter la Suisse en I889 pour « ce Paris, carrefour du monde », " [son] centre intellectuel, l'aimant auquel [il a] l'intellect orbité $»^{33}$ et où il compte "surveiller [son] bouquin [Egyptiacque] et [se] démener pour percer à tout prix ${ }^{34}$ puis la Roumanie dès I890 : "Je me hâtai de brûler mes vaisseaux. [...] N'importe quoi en Roumanie, mais la Roumanie ${ }^{35}$.

\section{William Ritter et les « petits messieurs corrects, distingués et venimeux de la littérature romande $»^{36}$. Une réception ambivalente}

En cette fin de $\mathrm{XIX}^{\mathrm{e}}$ siècle, le champ littéraire apparaît remarquablement homogène : la " chapelle "de tête" de la littérature romande sera plutôt libérale-conservatrice » et « le protestantisme se devra d'être affirmé » (Maggetti I995 :336), à l'instar de ce qu'Alain Clavien a appelé la « bande des quatre » (critiques littéraires, " héritiers », fils d'intellectuels, licenciés en droit ou en lettres et faisant carrière en Suisse essentiellement), dont la figure tutélaire (aux côtés de Monnier, Seippel et Vallette) est le Neuchâtelois Philippe Godet (I850-I922) (Clavien I993 : 55, 50). Actif au sein des plus importantes revues et des grands journaux - la Bibliothèque universelle ou La Suisse libérale de Neuchâtel -, mais aussi écrivain et professeur, Godet occupe une position centrale, et ses jugements font autorité. S'il mène alors une inlassable croisade pour la professionnalisation du métier d'écrivain (ce avec quoi Ritter ne peut qu'être d'accord, lui qui déplore le sort des versificateurs qui «se sont étiolés dans l'obscurité de professorats ridiculement bridés ${ }^{37}$ et qui désire vivre uniquement pour l'art mais surtout de son art) et qu'il milite pour un plus grand soin apporté à la langue et au style, il réaffirme la priorité accordée à la morale. « Notre littérature est essentiellement didactique et militante ", écrit-il dans son Histoire littéraire de la Suisse française en I895. Ces positions, largement majoritaires, vont fortement influencer l'accueil

32 L.a.s. de WR à GdR, s.l., 2I.II.1903, Fonds Reynold, corr. aut. 184, ALS, Berne.

33 WR, carnet 35, 17.09.1889, Fonds Ritter, ALS, Berne.

34 WR, carnet 36, 22.II.I889, Fonds Ritter, ALS, Berne.

35 WR, «Préface » aux «Lettres écrites de Castel Pelesch », Fonds Ritter, ALS, Berne, pp. 9-Io.

36 WR, "Causerie littéraire. Livres récents » (à propos de Roméo et Juliette au village de Gottfried Keller), Le National suisse, 22.II.I895.

37 WR, «Un poète suisse. Louis Duchosal », op. cit. 
des textes de Ritter et laisser apparaître plusieurs lignes de fracture. Avant d'entrer dans le détail des recensions ${ }^{38}$, il faut relever la grande quantité d'articles parus et la quasi-unanimité des chroniqueurs pour célébrer son talent littéraire et louer ses compétences musicologiques, sa vaste érudition ou ses descriptions du littoral neuchâtelois. Si la bonne réception parisienne n'est guère étonnante (les commentaires sont globalement élogieux de la part des écrivains amis, comme Judith Gautier dans Le Rappel, Rachilde pour le Mercure de France, Yvanhoé Rambosson à La Plume), les opinions en Suisse sont plus ambivalentes et transcendent le clivage entre journaux neuchâtelois et confédérés. Au cœur du débat : les attaques contre les mœurs suisses (en particulier au début d'Ægyptiacque); des scènes jugées trop sensuelles et les passions dépravées des personnages; le style, fait de mots rares, de néologismes, de formules chantournées, et de libertés prises avec la ponctuation. Sans surprise, les critiques et journaux libéraux-conservateurs se montrent les plus sévères. Alors que la Gazette de Lausanne préfère garder le silence sur Ægyptiacque («si nous n'en avons rien dit, c'est que nous n'en pouvions faire qu'une critique $\left.{ }^{39}\right)$, La Suisse libérale et la Bibliothèque universelle ne mâchent pas leurs mots. Godet critique ainsi le choix d'un « sujet scabreux, qui devait appeler des peintures d'une crudité révoltante » et déplore l'influence de Péladan et de Barbey d'Aurevilly : «[Ritter] a appris à leur école à unir, dans une combinaison raffinée et perverse, je ne sais quelle affectation de mysticisme et je ne sais quelle obsession de sensualité ; cela forme un mélange équivoque et malsain, plus dangereux encore que les brutalités populacières d'un Zola. » Quant au style, s'il reconnaît à Ritter un talent réel, il le trouve encore «très inégal, compliqué d'imitation, de prétentions et de parti pris d'école ", et incite le jeune écrivain à simplifier et à «purifier» sa langue. L'emportement de Ritter contre Neuchâtel lui apparaît enfin comme le caprice d'« un enfant en colère occupé à piétiner ses jouets ». le Journal de Genève, par la voix de Paul Seippel (I858-1926), se montre moins tranchant certes, mais fait preuve de beaucoup d'ironie vis-à-vis des « belles illusions de la jeunesse », de la «ferveur de néophyte » et de l'« outrance toute juvénile » de son style, tout en reconnaissant qu'il s'agit peut-être du " début littéraire le plus intéressant auquel nous ayons assisté depuis longtemps dans la Suisse romande ». Quant à L'Impartial, journal indépendant qui a refusé la collaboration de Ritter en I89ı pour cause de divergence à propos du rôle moral attribué à l'art ${ }^{40}$, il regrette que l'écrivain s'attarde à

38 Regroupées par Ritter dans deux carnets intitulés « Gloriolicule I Ægyptiacque » et « Gloriolicula II Âmes blanches » et d'où proviennent toutes les citations suivantes, Fonds Ritter, ALS, Berne.

39 L.a.s. de la Gazette de Lausanne (Édouard Secretan) à WR, Lausanne, 08.02.1893, Fonds Ritter, ALS, Berne.

40 L.a.s. de L'Impartial (Edmond Beaujon) à WR, La Chaux-de-Fonds, 29.0I.I89I, Fonds Rit- 
« dépeindre des gens qui n'ont que des instincts » et qui se laissent dominer par leurs passions, et évoque les « trop nombreux néologismes et une tendance un peu décadente » qui gâtent son écriture. Ritter encaissa non sans peine ces reproches (de même que l'interdiction de vente d'Ægyptiacque dans les gares, prononcée par la maison Hachette) et c'est bien de Godet et de ses conceptions littéraires dont il se moque en parlant de " pierre-à-niton dans la baie de ses tranquilles eaux suissardes dont il a si longtemps marqué de sa médiocrité l'étiage $»^{4}$. Écrivant en 1886 déjà que Godet « mord les mol-

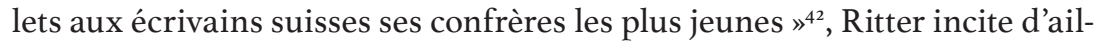
leurs son cadet, Gonzague de Reynold, à faire fi de l'opinion du juge des lettres romandes en 1903 : "Vous croyez donc que cela existe vraiment, Godet, Autier, tout cela! Je rêve pour vous que vous deveniez un d'Aurevilly fribourgeois et vous me répondez presque que vous vous souciez du suffrage de ces gens-là pour vous... et ce qui pis est pour moi ! » ${ }^{43}$ Laccueil fut en revanche beaucoup plus favorable de la part des journaux radicaux et socialistes ( $L a$ Sentinelle de La Chaux-de-Fonds use même d'un ton messianique - « un vrai, un réel écrivain nous est né » - et estime que les Neuchâtelois de la ville « méritent qu'on leur dise de temps à autre des vérités », parlant même d'une " foule de médiocres blessés dans leur vanité»). Il faut rappeler ici que si les libéraux et les radicaux se rapprochent politiquement face à la montée du socialisme, leurs " antagonismes se transportent sur le terrain littéraire " (Clavien, Vallotton 2007 : 35I). Le National suisse, quotidien radical de La Chaux-de-Fonds, se montre par exemple bienveillant vis-à-vis de Ritter (dont il publia des textes) en parlant d'Egyptiacque comme d'un « livre qui classe son auteur au premier rang des écrivains romands et des bons écrivains français ». Quant à Virgile Rossel (I858-1933), critique littéraire radical et adversaire de Godet, il accorde quelques lignes élogieuses à Ritter dans son Histoire de la littérature française hors de France (I895) et, s'il regrette les « brutalités de plume » et l'« affectation moderniste » d'Egyptiacque, il estime que les débuts de Ritter, qui « n'est nullement dans la tradition romande », méritent d'être salués : « Toujours est-il que l'originalité n'étant pas denrée commune sur le marché littéraire romand, j'ai jugé convenable de signaler à notre public un jeune écrivain qui est bien armé pour la course à la gloire. » Le changement de point de vue est significatif : alors que les journaux les plus sévères à l'égard de Ritter se livrent principalement à une analyse interne, ou s'en écartent uniquement pour déconsidérer les modèles français que le jeune écrivain s'est choisis, les critiques qui lui sont globalement favorables insistent sur la place de cette œuvre dans le

\footnotetext{
ter, ALS, Berne.

4I C.a.s. de WR à GdR, Prague, I2.I0.1904, Fonds Reynold, corr. aut. I84, ALS, Berne.

42 WR, carnet I8, 22.02.I886, Fonds Ritter, ALS, Berne.

43 C.a.s. de WR à GdR, Munich, 24.II.I903, Fonds Reynold, corr. aut. I84, ALS, Berne.
} 
paysage suisse francophone et sur son aspect novateur - non sans profiter de décocher quelques flèches contre le milieu littéraire, comparé à une «petite mare tranquille où il y a quelques grenouilles et beaucoup de crapauds » (Duchosal) ou dans laquelle «barbotteraient des oies» (Biolley). La parution des livres de Ritter est ainsi l'occasion, pour les personnalités en marge des cercles dominants, de dénoncer le « sirop anodin de nos faiseurs » (Duchosal), les «plumitifs à l'eau de rose et au sirop d'orgeat » (Biolley), ainsi que les conditions de vie offertes aux écrivains en Suisse. "Les artistes peuvent difficilement vivre dans notre atmosphère » écrit encore Duchosal, qui compare la Suisse à une "maison fermée qui a besoin d'être aérée et qu'on pousse les volets ", tandis que Bachelin note que Ritter " est encore plus connu et apprécié au-delà de nos frontières qu'en de-ça. Toujours la vieille histoire : nul n'est prophète dans son pays. "Son ancien professeur n'hésite d'ailleurs pas à en faire un porte-étendard : «il plaide et parle moins pour lui en particulier que pour les artistes en général, ses frères-de-croix de l'idéal, qui ont tous eu à souffrir ou peu ou prou de l'esprit bourgeois. »

\section{«Pauvre Revue de Genève ! Je lui en ai toujours gardé un souvenir ému et reconnaissant, d'avoir accueilli la première de mes lourdes proses d'écolier romantique! $»^{44}$ William Ritter dans quelques revues suisses}

Les organes de presse francophones qui accueillirent Ritter au tournant du siècle peuvent être classés en quatre groupes : en France et en Belgique, les périodiques d'obédience symboliste comme La Plume, La Revue Blanche et le Mercure de France ou Le Magasin littéraire et scientifique et Durendal ; en Suisse, les quotidiens neuchâtelois (plutôt de tendance radicale, comme Le National suisse d'Henri Wolfrath, avec la parution de son tout premier article en janvier I886) et fribourgeois (catholiques, comme La Liberté) ; et enfin les "revues de jeunes" (La Revue de Genève de Duchosal qui publia son premier texte, "Le Damné », en juin I886, mais aussi Les Pages littéraires ou La Montagne) et les "jeunes revues" (Le Foyer domestique, La Semaine littéraire), objets du présent chapitre. Les choix éditoriaux de Ritter, mais aussi l'attitude adoptée à son endroit par les directeurs de revues, sont autant dictés par le parcours erratique de l'écrivain (entre nécessité de gagner sa vie, voyages à l'étranger et rencontres personnelles) que par le positionnement de ces périodiques dans le champ littéraire des années I890 et par la réputation (sulfureuse) du critique neuchâtelois.

44 L.a.s. de WR à Louis Duchosal, Monruz (Neuchâtel), 09.03.I89I, Correspondance Louis Duchosal, CH BGE Ms. fr. 2989, f. 250, Genève. 
Revues « ouvertes ", pour reprendre la typologie proposée par Clavien, Le Dinh et Vallotton (I993: I2), Les Pages littéraires, lancées en I894 par Louis Moriaud (né en I869) et Valentin Grandjean (I872-1944), et La Montagne, fondée notamment par Grandjean en I896 suite à un conflit entre les deux directeurs, se distinguent par leur volonté d'offrir une tribune aux nouvelles plumes tout en affirmant - modestement, et au point de vue théorique - leur ambition de renouveler la littérature romande. À cet égard, Ritter fait en quelque sorte office de "parrain" : plus vraiment débutant, il appartient toutefois encore à la jeune génération et ses œuvres semblent douées d'un fort potentiel subversif. Moriaud souhaite par exemple une recension « polémique $»^{45}$ sur l'étude que Ritter consacra à Böcklin et loue en particulier les premières pages d'Ægyptiacque, "Genève [ressemblant] tellement à Neuchâtel ! $»^{46}$ Daniel Baud-Bovy (I870-1958), cofondateur de La Montagne, évoque, lui, une "manifestation un peu de guerre civile, plus que d'esthétique ${ }^{47}$ à propos du conflit opposant Ritter à Godet sur l'appréciation des peintures de Paul Robert ${ }^{48}$ et compare l'attitude de Ritter à celle de son père, le peintre Auguste Baud-Bovy, qui « savait exciter les haines sourdes » de ses "doux compatriotes " $^{49}$. L'écrivain neuchâtelois rencontra donc un accueil très favorable dans ces "revues de jeunes" nouvellement créées. Les Pages littéraires font ainsi paraître quatre articles de Ritter : aux côtés de critiques artistiques figurent une biobibliographie de Bachelin et un texte plus personnel (« Nocturne viennois »), tandis que La Montagne publie Ritter à deux reprises (à propos de Storm van's Gravesande et de Hans Sandreuter). Mais plus que le nombre et la nature des textes publiés, c'est bien la rhétorique des directeurs de revue qu'il convient de relever. Essuyant de nombreux refus des grands éditeurs de presse, Ritter fait cette fois l'objet de sollicitations de la part de ses jeunes compatriotes. «Votre nom, Monsieur, s'est trouvé immédiatement sous notre plume, et nous venons vous demander l'autorisation de vous placer à l'avant-garde de notre phalange " ${ }^{50}$, lui écrit Moriaud en I892, alors qu'il désire fonder un périodique sous le titre " La Revue de Genève ». Deux ans plus tard, le lancement des Pages littéraires est l'occasion de rappeler à Ritter sa promesse de collaboration et de louer l'écrivain : "nous aimons tous et nous admirons le talent de l'auteur d'Ægyptiaque "5I, allant même jusqu'à dire, non sans flatterie, qu'« aucune collaboration ne

45 C.a.s. de Louis Moriaud à WR, Genève, 02.07.I895, Fonds Ritter, ALS, Berne.

46 L.a.s. de Louis Moriaud à WR, Genève, 27.09.1894, Fonds Ritter, ALS, Berne.

47 L.a.s. de Daniel Baud-Bovy à WR, Blonay, 04.II.I894, Fonds Ritter, ALS, Berne.

48 WR, «Trois journées d'art à Neuchâtel. I. M. Paul Robert » et «Les tableaux de M. Paul Robert ", Le National suisse, 25.04 et 06.05.1894.

49 L.a.s. de Daniel Baud-Bovy à WR, Genève, 28.II.I899, Fonds Ritter, ALS, Berne.

50 L.a.s. de Louis Moriaud à WR, Genève, 29.07.I892, Fonds Ritter, ALS, Berne.

5I L.a.s. de Louis Moriaud à WR, Genève, II.07.I894, Fonds Ritter, ALS, Berne. 
pouvait [leur] être plus agréable $»^{52}$. Grandjean sollicitera également Ritter, à la plume « toujours si originale et si artiste " ${ }^{53}$ et dont le nom « est celui d'un des plus vaillants parmi la phalange de nos jeunes écrivains "54. À en croire les lettres conservées aux ALS, Ritter semble s'être montré plutôt favorable aux initiatives lancées par ses deux cadets (allant jusqu'à leur donner des conseils). Son éloignement progressif résulte donc bien plus vraisemblablement de causes extra-littéraires : outre son départ de Suisse (rappelons qu'il vit à Vienne durant la majeure partie de la décennie I890), ses choix éditoriaux paraissent moins dictés par des critères idéologiques et esthétiques que par des contraintes matérielles. Pour celui qui a formulé très tôt le vœu de gagner sa vie comme écrivain et comme critique d'art, sans ressources financières familiales ni réelle profession d'appoint, il est essentiel de voir ses contributions rétribuées. Les moyens modestes dont disposent les deux revues genevoises (qui plus est dépourvues de capital symbolique "compensatoire") sont alors sans doute un obstacle de taille à la participation de Ritter sur le long terme.

Les rapports furent sensiblement différents avec La Semaine littéraire, lancée en I893 par Louis Debarge, et Le Foyer domestique, porté sur les fonds baptismaux par la maison Attinger en i888. Alliant conservatisme (le profil de ses fondateurs, Jules Sandoz et Carl Russ-Suchard, en témoigne) et modernité (à propos de la place donnée aux femmes ou du soin accordé à l'illustration), Le Foyer domestique publie notamment des «feuilletons et nouvelles littéraires de bonne tenue souvent rédigés par des auteur(e)s du cru » (Pavillon \& Vallotton I993 : 48), tandis que La Semaine littéraire, tout en restant passablement prudente vis-à-vis des innovations esthétiques, se montre plus ouverte que la Bibliothèque universelle et mène « une politique souple et conciliante qui lui permet de réunir dans ses sommaires les représentants des différentes chapelles littéraires » (Revaz 20I5 : 43I). "Jeunes revues", Le Foyer domestique et La Semaine littéraire, tout en possédant une assise financière et une diffusion considérablement plus importantes, doivent faire preuve de prudence pour maintenir un équilibre précaire, entre impératifs économiques, qualité littéraire et goût du public romand. La collaboration avec Ritter s'en ressent. S'il publie beaucoup (une quarantaine de textes environ par revue) et des articles fort divers : on y trouve en effet autant des critiques artistiques, que des récits autobiographiques neuchâtelois, des relations de voyage ( Second séjour au Monténégro ») et des œuvres en prose («Les quatre saisons », "Sur le chemin de Maria Zell » et « La douce compassion de la mer et du ciel»), la liberté dont il bénéficiait dans les "revues de jeunes" n'est ici plus de mise. La grande majorité des propositions émanent

52 C.a.s. de Louis Moriaud à WR, Genève, 20.09.1894, Fonds Ritter, ALS, Berne.

53 L.a.s. de Valentin Grandjean à WR, Cannes, 07.03.1895, Fonds Ritter, ALS, Berne.

54 L.a.s. de Valentin Grandjean à WR, Genève, 06.03.1896, Fonds Ritter, ALS, Berne. 
cette fois-ci de Ritter, qui cherche à placer ses articles contre rétribution. $\mathrm{Si}$ la revue neuchâteloise doit lui rappeler à plusieurs reprises la relative modestie de ses moyens, le malentendu éclate en novembre I896, face aux (probables) récriminations de Ritter : «vous nous avez toujours montré tant de bienveillance que les questions financières semblaient écartées au second plan. ${ }^{55}$ En outre, le type d'article lui est le plus souvent suggéré (Debarge réclame par exemple une nouvelle avec « un sujet simple, capable d'intéresser un grand nombre de lecteurs et d'éviter n'est-ce pas ce qui peut nuire à une publication jeune encore en pays romand $»^{56}$, tandis qu'Attinger lui demande " une bluette bien honnête » ou l'une de ses "piécettes virginales " ${ }^{57}$ ) et plusieurs de ses textes seront finalement refusés pour des raisons morales - quand on ne se contente pas d'exiger la suppression des épithètes les plus problématiques. Autant Debarge qu'Attinger rappellent ainsi plusieurs fois à Ritter la nécessité de ménager leur public, composé notamment, pour Le Foyer, des " petits bourgeois " et des " ouvriers aisés " ${ }^{8}$. "Là aussi on me tolère même de temps en temps, si je promets de me comporter comme un jeune homme bien élevé, et si je reviens de très loin $»^{59}$, ironise-t-il d'ailleurs avec malice dans un article du National suisse présentant la revue neuchâteloise. Synonyme d'originalité et d'ouverture, gage également d'une certaine qualité littéraire (Attinger en fait même l'un de ses « collaborateurs de marque $\left.{ }^{60}\right)$, l'écrivain fait aussi figure de bombe à retardement, que les éditeurs doivent manier avec précaution...

\section{William Ritter : un décadent (en) suisse à l'avant-garde?}

Une question conclusive volontairement provocante tant la décadence, telle qu'elle a pu être théorisée en France, aussi bien d'un point de vue littéraire (par Jean de Palacio par exemple, dont La Décadence. Le mot et la chose est paru en 20II) que sous l'angle plus largement sociopolitique (avec Décadence fin de siècle de Michel Winock en 2017), ne s'applique guère à la Suisse romande et a fortiori à sa littérature. Cela tient évidemment à l'histoire politique et culturelle radicalement différente des deux pays : le naturalisme, contre lequel se soulèvent les symbolistes et les décadents français, n’a pas fait d'émules de ce côté-ci du Jura tandis que la culture politique helvétique (avec la Constitution de I848, l'avènement du radicalisme et la prédomi-

55 L.dact.s. de Victor Attinger à WR, Neuchâtel, 02.II.I896, Fonds Ritter, ALS, Berne.

56 L.a.s. de Louis Debarge à WR, Genève, 30.0I.1895, Fonds Ritter, ALS, Berne.

57 L.dact.s. de Victor Attinger à WR, Neuchâtel, 07.I2.1892 et 08.I0.I894, Fonds Ritter, ALS, Berne.

58 L.dact.s.. de Victor Attinger à WR, Neuchâtel, 2I.II.I895, Fonds Ritter, ALS, Berne.

59 WR, «Périodiques illustrés », in Le National suisse, 06.I2.I895.

60 C.dact.s. de Victor Attinger à WR, Neuchâtel, 30.06.1898, Fonds Ritter, ALS, Berne. 
nance du protestantisme) paraît bien éloignée des révolutions qui ont secoué l'Hexagone durant le long XIX ${ }^{\mathrm{e}}$ siècle (de la chute de l'Ancien Régime en I789 à l'instauration de la Troisième République, en passant par la défaite de Sedan et la montée du socialisme). Si le concept même de "décadence" a connu des fortunes diverses dans les pays limitrophes de la France, les champs littéraires suisse et français ne se recoupent pas (bien au contraire même !) et, si "décadence" (en) suisse il y a eu, cela n’a pu avoir lieu que sous la forme d'une adaptation aux réalités helvétiques et dans des cas extrêmement limités, à commencer par le "premier Ritter", celui d'Egyptiacque en particulier. Sa mise en scène romanesque d'une Suisse aristocratique et patricienne, son style "byzantin"6r et son goût pour le néologisme et les mots rares, sa défense d'un catholicisme teinté de mysticisme, ses saillies antisémites et antijuives, enfin son dandysme, son élitisme et son rejet du progrès peuvent effectivement le rapprocher des écrivains français décadents les plus célèbres ou, par certains aspects, de ceux qu'Antoine Compagnon a appelé les « antimodernes ». L'œuvre "décadente" de Ritter paraît en revanche hautement subversive en Suisse romande, où le terme, sous la plume des principaux critiques, gardiens de l'orthodoxie littéraire romande, faisait alors plutôt figure d'épouvantail pour déconsidérer une littérature et des écrivains désireux de s'affranchir des normes établies, comme en témoignent les propos du puissant directeur de la Bibliothèque universelle en I886 : "Si les décadents suisses vont à Paris, c'est parce que la Suisse ne leur offre pas un terrain favorable : là où est le cadavre, là s'assemblent les vautours. " ${ }^{62}$ Isolés au sein de la production livresque de l'époque, soutenus uniquement par des personnalités en marge des cercles dominants (essentiellement marquées par le symbolisme), les romans de Ritter n'ont guère donné lieu à des études approfondies. La qualité littéraire inégale de ses textes, la masse gigantesque de documents d'archives déposés dans trois institutions patrimoniales différentes (Berne, Neuchâtel et La Chauxde-Fonds) et sa rupture autant géographique que littéraire avec son pays natal expliquent peut-être en partie le manque de considération dont il a été victime jusqu'à récemment. L'hypothèse développée dans ces pages et soutenue par Gonzague de Reynold en I960 - « cet écrivain [...] fut le premier à sortir les lettres romandes du conformisme et du moralisme où elles

6I Le terme apparaît sous la plume de Ritter, pour parler du style de son « Damné » : « Pour celui-ci je compte châtier mon style avec un byzantinisme à la Flaubert, il faut que ce soit un chef-d'œuvre » (2I.I2.I885, carnet I8) ou à propos de la littérature moderne, dans un passage qui suit directement sa lecture de À Rebours de Huysmans : « Tout le bas-empire byzantin des jeunes de la littérature moderne actuelle me plaît par son byzantinisme de forme et m'énerve par son fonds trop bas-empire » (17.0I.I886, carnet I8).

62 Lettre d'Édouard Tallichet à Philippe Godet, 22.06.1886, citée dans Revaz \& Vallotton (2015: 430). 
mijotaient ${ }^{63}$ - mériterait pourtant d'être étayée, voire confirmée, par une analyse interne plus fine de ses romans. Tache aveugle de l'histoire de la littérature en Suisse romande, l'œuvre du jeune Ritter, qui a pu, dans une certaine mesure, préparer le terrain aux avant-gardes littéraires romandes du début du $x^{\mathrm{e}}$ siècle, ne serait-ce que dans l'émancipation de la morale et l'attention magnifiée à la langue, se situe peut-être au croisement de deux récits historiques aujourd'hui bien documentés : la constitution du champ littéraire romand dès I850 et l'avènement de la nouvelle génération qui se fédère dès 1904 autour des Pénates d'argile et de La Voile latine.

\section{Bibliographie}

Clavien, Alain, Les Helvétistes. Intellectuels et politique en Suisse romande au début du siècle, Lausanne, Éditions d'en bas, I993.

-. «Les revues suisses et la France : Paris si loin, trop proche », in Jacqueline Pluet-Despatin, Michel Leymarie et Jean-Yves Mollier (dirs.), La Belle Époque des revues I880-I9I4, Paris, Éditions de l'IMEC, 2002, pp. 335-345.

Clavien, Alain \& Vallotton, François, «Les supports de la critique littéraire en Suisse romande : grandes revues, "variétés" et suppléments littéraires. I830-1960 ", Cahiers de l'Association internationale des études françaises, $\mathrm{n}^{\circ}$ 59, mai 2007, pp. 337-355.

Clavien, Alain, Le Dinh Diana \& Vallotton, François, «Jalons pour une histoire à faire : les revues romandes, I880-I9I4 ", Histoires de revues, Les Annuelles, $\mathrm{n}^{\circ}$ 4, I993, pp. 7-27.

Maggetti, Daniel, L'Invention de la littérature romande I830-I9Io, Lausanne, Éditions Payot, 1995.

Michaud, Marius, «William Ritter vu par ses contemporains suisses : "amateur d'Europe centrale" et contempteur de son pays natal », in Actes du colloque "L'Europe centrale en amateur. William Ritter (I867-I955) », 2I-22 novembre 2008, Paris, Centre interdisciplinaire de Recherches centre-européennes, www.circe.paris-sorbonne.fr/spip.php?articlei94.

-. "Une amitié tumultueuse : Gonzague de Reynold et William Ritter (I903-I955) ", in Clio dans tous états, Gollion, Infolio Édition, 2009, pp. 53I546.

Pavillon, Monique \& Vallotton, François, " "Le Foyer domestique, Journal pour la famille” I888-I905 : stratégies éditoriales, enjeux sociaux, politique des genres ", Histoires de revues, Les Annuelles, n 4, I993, pp. 46-7I.

63 GdR, Mes Mémoires, Genève, Éditions Générales, 1960, tome 2, p. 308. 
Revaz, Gilles \& Vallotton, François, « La vie littéraire au cours de la seconde moitié du XIX ${ }^{\mathrm{e}}$ siècle ", in Histoire de la littérature en Suisse romande, Carouge-Genève, Éditions Zoé, 2015, pp. 425-444.

William Ritter (I867-I955) au temps d'une autre Europe, Fernand Donzé, Caroline Calame et Edmond Charrière (dirs.), Nouvelle Revue neuchâteloise, $n^{\circ} 6 \mathrm{I}, \mathrm{I} 6^{\mathrm{e}}$ année, printemps 1999. 